\title{
An interface problem with singular perturbation on a subinterval
}

Feng Xie*

"Correspondence: fxie@dhu.edu.cn Department of Applied Mathematics, Donghua University, Shanghai, 201620, P.R. China

\begin{abstract}
In this paper we investigate an interface problem with singular perturbation on a subinterval. We first establish a lemma of lower and upper solutions which is an extension of the classical theory of lower and upper solutions. Based on the basic lemma we obtain the existence of a solution to the proposed problem, and the asymptotic behavior of solution as the singular perturbation parameter $\varepsilon \rightarrow 0^{+}$as well.
\end{abstract}

MSC: 34E10; 34A36; 34B15

Keywords: lower and upper solutions; singular perturbation; interface conditions

\section{Introduction}

Interface problems, like coupled elliptic-hyperbolic or parabolic-hyperbolic problems with discontinuous coefficients, arise in many fields, such as material sciences, fluid-solid interactions. If an interface problem is confined in a one dimensional domain, one gets a boundary value problem of ordinary differential equations with interface conditions. For example, in [1] de Falco and O'Riordan considered a one dimensional metal-oxidesemiconductor structure which is modeled by a two-point interface boundary value problem with singular perturbation. Recently, interface problems have attracted much attention as regards both theoretical and numerical aspects; see for instance [2-5] and references therein.

In [6] Aguilar and Lisbona investigated $C^{1}$-smooth solution of the following interface boundary value problem with singular perturbation:

$$
\left\{\begin{array}{l}
-\lambda_{\varepsilon}\left(\mu(w) w^{\prime}\right)^{\prime}+K(w)^{\prime}+b(x, w)=0, \quad x \in(0,1), \\
w(0)=w(1)=0
\end{array}\right.
$$

where $\lambda_{\varepsilon}$ is the piecewise constant function of the form

$$
\lambda_{\varepsilon}=\left\{\begin{array}{ll}
1, & x \in(0, c), \\
\varepsilon, & x \in(c, 1),
\end{array} \quad c \in(0,1)\right.
$$

and the functions $\mu \in C^{2}(\mathbb{R}), b \in C^{2}([0,1] \times \mathbb{R}), K \in C^{2}(\mathbb{R})$ satisfy

$$
\mu(w) \geq \mu_{0}>0, \quad b_{w}(x, w) \geq v>0, \quad K \text { strictly monotone. }
$$

C) 2014 Xie; licensee Springer. This is an Open Access article distributed under the terms of the Creative Commons Attribution License (http://creativecommons.org/licenses/by/4.0), which permits unrestricted use, distribution, and reproduction in any medium, provided the original work is properly credited. 
This kind of problem arises from some simplified physical models such as the infiltration process in an inhomogeneous soil [7]. In [6], by using inverse-monotone operator theorems, the authors proved that the unique $C^{1}$-smooth solution converges almost everywhere to the solution of the corresponding reduced problem as $\varepsilon \rightarrow 0^{+}$.

In physical problems, several typical interface conditions, such as perfect contact, flux jump, and thermal resistance, are often encountered. Hence, it is interesting and of significance to study the problem (1) with general interface conditions. In the present paper, as a natural generalization, we consider the following boundary value problem with interface conditions:

$$
\left\{\begin{array}{l}
\lambda_{\varepsilon} y^{\prime \prime}=g(x, y) y^{\prime}+f(x, y), \quad x \in(0, c) \cup(c, 1), \\
y(0)=y(1)=0, \\
{[y](c)=\alpha, \quad\left[y^{\prime}\right](c)=\beta,}
\end{array}\right.
$$

where we denote by $[y](c) \equiv y\left(c^{+}\right)-y\left(c^{-}\right)$the jump of $y$ at the point $c$. If we set $y=\int_{0}^{w} \mu(t) d t$ in (1), the problem (1) becomes

$$
\left\{\begin{array}{l}
\lambda_{\varepsilon} y^{\prime \prime}=\frac{K^{\prime}\left(w^{-1}(y)\right)}{\mu\left(w^{-1}(y)\right)} y^{\prime}+b\left(x, w^{-1}(y)\right), \quad x \in(0,1), \\
y(0)=y(1)=0
\end{array}\right.
$$

which is a special form of (2) with $\alpha=\beta=0$.

In Section 2, we establish first a lemma of low and upper solutions for the problem (2), which is an extension of classical theory of lower and upper solutions. Lower and upper solutions theorems for $C^{1}$-smooth solutions of two-point second-order boundary value problems with discontinuous coefficients have been established in [8] where $W^{2,1}$ solutions ( $C^{1}$-smooth certainly) are considered. However, the theory of lower and upper solutions for boundary value problems with general interface conditions has not been formulated, to our knowledge.

In Section 3, based on the basic lemma established in Section 2 we analyze the asymptotic behavior of solution to the problem (2) in everywhere sense. The original problem can be viewed as the coupling of the left problem and the right singular perturbation problem satisfying the jump interface conditions. The solution of the right problem exhibits generally a boundary layer at either end, which depends on the sign of $g(x, y)($ see $[9,10]$ for instance). Thereby two cases should be distinguished. We prove that under suitable conditions the problem (2) has a solution whose asymptotic behavior is fully described as $\varepsilon \rightarrow 0^{+}$on the whole interval $[0,1]$. A simple linear example as an illustration is presented at the end.

Throughout this paper, we assume

(H1) The functions $f(x, y)$ and $g(x, y)$ are $C^{1}$-smooth, and $f_{y}^{\prime}(x, y) \geq v>0$ on $[0,1] \times \mathbb{R}$.

\section{Lower and upper solutions lemma}

For $j=1,2$, let $Q^{j}[0,1]$ be a vector space of functions $u(x)$ defined on $[0,1]$ satisfying

$$
\left.u(x)\right|_{[0, c]} \in C^{j}[0, c],\left.\quad u(x)\right|_{[c, 1]} \in C^{j}[c, 1],
$$

where $u(x)$ is double-valued at $x=c$. 
Definition 1 A function $\Phi(x) \in Q^{2}[0,1]$ is called a lower solution of the problem (2) if

$$
\begin{aligned}
& \lambda_{\varepsilon} \Phi^{\prime \prime}(x) \geq g(x, \Phi(x)) \Phi^{\prime}(x)+f(x, \Phi(x)), \quad \text { for } x \in(0, c) \cup(c, 1), \\
& {[\Phi](c)=\alpha, \quad\left[\Phi^{\prime}\right](c) \geq \beta,} \\
& \Phi(0) \leq 0, \quad \Phi(1) \leq 0 .
\end{aligned}
$$

A function $\Psi(x) \in Q^{2}[0,1]$ is called an upper solution of the problem (2) if

$$
\begin{aligned}
& \lambda_{\varepsilon} \Psi^{\prime \prime}(x) \leq g(x, \Psi(x)) \Psi^{\prime}(x)+f(x, \Psi(x)), \quad \text { for } x \in(0, c) \cup(c, 1), \\
& {[\Psi](c)=\alpha, \quad\left[\Psi^{\prime}\right](c) \leq \beta,} \\
& \Psi(0) \geq 0, \quad \Psi(1) \geq 0 .
\end{aligned}
$$

Lemma 1 Assume that $\Phi$ and $\Psi$ are lower and upper solutions of the problem (2) such that $\Phi \leq \Psi$. Then the problem (2) has at least one solution $y \in C^{2}([0, c) \cup(c, 1])$ such that for all $x \in[0,1]$

$$
\Phi(x) \leq y(x) \leq \Psi(x)
$$

Proof Let us define the following modifications of the functions in the right hand side of (2):

$$
\bar{F}\left(x, y, y^{\prime}\right)= \begin{cases}F\left(x, \Phi(x), y^{\prime}\right)+\frac{y-\Phi(x)}{1+|y-\Phi(x)|}, & \text { if } y<\Phi(x), \\ F\left(x, y, y^{\prime}\right), & \text { if } \Phi(x) \leq y \leq \Psi(x), \\ F\left(x, \Psi(x), y^{\prime}\right)+\frac{y-\Psi(x)}{1+|y-\Psi(x)|}, & \text { if } y>\Psi(x)\end{cases}
$$

and

$$
H\left(x, y, y^{\prime}\right)= \begin{cases}\bar{F}(x, y,-N), & \text { if } y^{\prime}<-N \\ \bar{F}\left(x, y, y^{\prime}\right), & \text { if }-N \leq y^{\prime} \leq N \\ \bar{F}(x, y, N), & \text { if } y^{\prime}>N\end{cases}
$$

where $F\left(x, y, y^{\prime}\right)=g(x, y) y^{\prime}+f(x, y)-\lambda_{\varepsilon} y$, and $N>0$ is a large enough number.

Consider the modified problem

$$
\left\{\begin{array}{l}
\lambda_{\varepsilon} y^{\prime \prime}-\lambda_{\varepsilon} y=H\left(x, y, y^{\prime}\right), \quad x \in(0, c) \cup(c, 1) \\
y(0)=y(1)=0, \\
{[y](c)=\alpha, \quad\left[y^{\prime}\right](c)=\beta}
\end{array}\right.
$$

Using the method of variation of constants, we write the solution of (8) in the following form:

$$
y(x)=p(x)+ \begin{cases}\int_{0}^{c} \lambda_{\varepsilon}^{-1} G_{1}(x, s) H(\cdot, \cdot, \cdot) \mathrm{d} s+\int_{0}^{1} \lambda_{\varepsilon}^{-1} \Theta_{1}(x, s) H(\cdot, \cdot, \cdot) \mathrm{d} s, & x \in[0, c], \\ \int_{c}^{1} \lambda_{\varepsilon}^{-1} G_{2}(x, s) H(\cdot, \cdot, \cdot) \mathrm{d} s+\int_{0}^{1} \lambda_{\varepsilon}^{-1} \Theta_{2}(x, s) H(\cdot, \cdot, \cdot) \mathrm{d} s, & x \in[c, 1],\end{cases}
$$


where $H(\cdot, \cdot, \cdot)=H\left(s, y(s), y^{\prime}(s)\right)$ and

$$
\begin{aligned}
& G_{1}(x, s)=\frac{1}{W^{-}}\left\{\begin{array}{ll}
w_{1}(x) w_{c}(s), & x \leq s, \\
w_{1}(s) w_{c}(x), & s \leq x,
\end{array} \quad G_{2}(x, s)=\frac{1}{W^{+}} \begin{cases}w_{2}(s) w_{c}(x), & x \leq s, \\
w_{2}(x) w_{c}(s), & s \leq x,\end{cases} \right. \\
& \Theta_{1}(x, s)=\frac{e^{c-1}+e^{1-c}}{e-e^{-1}}\left[\frac{w_{2}(s)}{W^{+}}-\frac{w_{1}(s)}{W^{-}}\right] w_{1}(x), \\
& \Theta_{2}(x, s)=\frac{e^{c}+e^{-c}}{e-e^{-1}}\left[\frac{w_{2}(s)}{W^{+}}-\frac{w_{1}(s)}{W^{-}}\right] w_{2}(x), \\
& W^{-}=-2\left(e^{c}+e^{-c}\right), \quad W^{+}=2\left(e^{1-c}+e^{c-1}\right),
\end{aligned}
$$

while the functions

$$
\begin{aligned}
& w_{1}(x)=\left\{\begin{array}{ll}
e^{x}-e^{-x}, & x \in[0, c], \\
0, & x \in[c, 1],
\end{array} \quad w_{2}(x)= \begin{cases}0, & x \in[0, c], \\
e^{x-1}-e^{1-x}, & x \in[c, 1],\end{cases} \right. \\
& w_{c}(x)=e^{x-c}+e^{c-x}, \quad x \in[0,1]
\end{aligned}
$$

solve the homogeneous equation $y^{\prime \prime}-y=0$. The function

$$
p(x)= \begin{cases}\frac{\left(e^{c-1}+e^{1-c}\right) \alpha+\left(e^{1-c}-e^{c-1}\right) \beta}{2\left(e^{-1} e\right)}\left(e^{x}-e^{-x}\right), & x \in[0, c], \\ \frac{\left(e^{c}+e^{-c}\right) \alpha+\left(e^{-c}-e^{c}\right) \beta}{2\left(e^{-1}-e\right)}\left(e^{x-1}-e^{1-x}\right), & x \in[c, 1],\end{cases}
$$

is the unique solution of the problem

$$
\left\{\begin{array}{l}
y^{\prime \prime}-y=0, \quad x \in(0, c) \cup(c, 1), \\
y(0)=y(1)=0, \\
{[y](c)=\alpha, \quad\left[y^{\prime}\right](c)=\beta .}
\end{array}\right.
$$

The integral equation (9) defines an operator $T$ on $Q^{1}[0,1]$, that is, a Banach space endowed with the norm $\|y\|=\|y\|_{\infty}+\left\|y^{\prime}\right\|_{\infty}$. Since $H\left(x, y, y^{\prime}\right):[0,1] \times \mathbb{R}^{2} \rightarrow \mathbb{R}$ is uniformly bounded in $y \in Q^{1}[0,1]$, the set $T\left(Q^{1}[0,1]\right)$ is a relatively compact subset of $Q^{1}[0,1]$. Moreover, $T$ is continuous. Hence, it follows from the Schauder fixed-point theorem (see, for instance, [11]) that the boundary value problem (8) has a solution. Note that any solution of (8) which lies between $\Phi(x)$ and $\Psi(x)$ and satisfies $\left|y^{\prime}(x)\right|<N, x \in[0,1]$, is a solution of (2).

Noting that $F\left(x, y, y^{\prime}\right)$ satisfies a Nagumo condition with respect to $\Phi(x)$ and $\Psi(x)$, it follows that $\left|y^{\prime}(x)\right|<N, x \in[0,1]$ (see Theorems 7.33 and 7.34 in [12]). In what follows, we prove $\Phi(x) \leq y(x), x \in[0,1]$. Suppose, on the contrary, that the function $u(x)=\Phi(x)-y(x)$ has a positive maximum at some $x_{0} \in[0,1]$. From (5) we see $x_{0} \in(0,1)$. If $x_{0} \in(0, c) \cup(c, 1)$, then $u\left(x_{0}\right)>0, u^{\prime}\left(x_{0}\right)=0$, and $u^{\prime \prime}\left(x_{0}\right) \leq 0$. On the other hand,

$$
\begin{aligned}
\lambda_{\varepsilon} u^{\prime \prime}\left(x_{0}\right)= & \lambda_{\varepsilon} \Phi^{\prime \prime}\left(x_{0}\right)-\lambda_{\varepsilon} y^{\prime \prime}\left(x_{0}\right) \\
\geq & g\left(x_{0}, \Phi\left(x_{0}\right)\right) \Phi^{\prime}\left(x_{0}\right)+f\left(x_{0}, \Phi\left(x_{0}\right)\right)-g\left(x_{0}, \Phi\left(x_{0}\right)\right) y^{\prime}\left(x_{0}\right)-f\left(x_{0}, \Phi\left(x_{0}\right)\right) \\
& -\frac{y\left(x_{0}\right)-\Phi\left(x_{0}\right)}{1+\left|y\left(x_{0}\right)-\Phi\left(x_{0}\right)\right|}+\lambda_{\varepsilon}\left(\Phi\left(x_{0}\right)-y\left(x_{0}\right)\right) \\
> & 0,
\end{aligned}
$$


which contradicts $u^{\prime \prime}\left(x_{0}\right) \leq 0$. If $x_{0}=c$, then $u^{\prime}\left(c^{-}\right) \geq 0 \geq u^{\prime}\left(c^{+}\right)$. In view of (4), it follows that $u^{\prime}\left(c^{-}\right)=u^{\prime}\left(c^{+}\right)=0$, which implies $u^{\prime \prime}\left(c^{-}\right) \leq 0$ and $u^{\prime \prime}\left(c^{+}\right) \leq 0$. Letting $x_{0} \rightarrow c^{ \pm}$in (10) yields a contradiction. This shows that $\Phi(x) \leq y(x)$ for $x \in[0,1]$. In a similar way, we can prove that $y(x) \leq \Psi(x)$ for $x \in[0,1]$.

Therefore, the solution of (8) is also that of (2) and satisfies $\Phi(x) \leq y(x) \leq \Psi(x)$ for $0 \leq$ $x \leq 1$.

\section{Asymptotic estimates}

In this section, we investigate asymptotic behavior of solutions of (2) by constructing suitable pairs of lower and upper solutions. As in [6], we distinguish two cases, and consider the asymptotic behavior under the assumptions $(\mathrm{H} 2)$ and $\left(\mathrm{H}^{\prime}\right)$, respectively.

(H2) There exists a positive constant $\sigma_{0}$ such that $g(x, y) \leq-\sigma_{0}<0$ for $(x, y) \in[0,1] \times \mathbb{R}$.

$\left(\mathrm{H} 2^{\prime}\right)$ There exists a positive constant $\sigma_{1}$ such that $g(x, y) \geq \sigma_{1}>0$ for $(x, y) \in[0,1] \times \mathbb{R}$.

Case (I). Assume (H2).

We also assume the following.

(H3) The reduced problem

$$
g(x, y) y^{\prime}+f(x, y)=0, \quad y(1)=0
$$

has a solution $\psi(x) \in C^{2}[c, 1]$.

Generally, the right problem

$$
\left\{\begin{array}{l}
\varepsilon y^{\prime \prime}=g(x, y) y^{\prime}+f(x, y), \quad x \in(c, 1), \\
y(c) \text { given, } \quad y(1)=0
\end{array}\right.
$$

has a boundary layer at $x=c$. However, taking the interface condition $\left[y^{\prime}\right](c)=\beta$ into consideration, the solution of (11) must have no boundary layer at $x=c$. Thus we have

$$
y\left(c^{+}\right)=\psi(c)+\mathcal{O}(\varepsilon)
$$

Proposition 1 The left boundary value problem

$$
\left\{\begin{array}{l}
y^{\prime \prime}=g(x, y) y^{\prime}+f(x, y), \quad x \in(0, c) \\
y(0)=0, \quad y(c)=\psi(c)+\alpha
\end{array}\right.
$$

has a solution $y=\varphi(x) \in C^{2}[0, c]$.

Proof It is easy to verify that $\Phi=-C_{0}$ and $\Psi=C_{0}$ are a pair of lower and upper solutions of (12), where

$$
C_{0}=\max \left\{|\psi(c)+\alpha|, \frac{|f(x, 0)|}{v}\right\} .
$$

Theorem 1 Let the conditions (H1), (H2), and (H3) hold. Moreover, we assume that

$$
g_{y}^{\prime}(x, \varphi(x)) \varphi^{\prime}(x) \geq 0, \quad x \in(0, c)
$$


Then for sufficiently small $\varepsilon>0$ the boundary value problem (2) has a solution $y(x, \varepsilon)$ satisfying

$$
y(x, \varepsilon)= \begin{cases}\varphi(x)+\mathcal{O}(\varepsilon), & x \in[0, c], \\ \psi(x)+\mathcal{O}(\varepsilon), & x \in[c, 1] .\end{cases}
$$

Proof From the assumptions (H1) and (H3) it follows that there is a positive constant $M$ such that for sufficiently small $\varepsilon>0$

$$
\begin{aligned}
& \left|\psi^{\prime \prime}(x)\right| \leq M, \quad x \in[c, 1], \\
& \left|g_{y}^{\prime}(x, \psi(x)+v) \psi^{\prime}(x)+f_{y}^{\prime}(x, \psi+v)\right| \leq M, \quad(x, v) \in[c, 1] \times[-M \varepsilon, M \varepsilon] .
\end{aligned}
$$

We construct the barrier functions as follows:

$$
\begin{aligned}
& \Phi(x)= \begin{cases}\varphi(x)-\gamma_{1} \varepsilon, & x \in[0, c], \\
\psi(x)-\gamma_{2} \varepsilon e^{-\sigma_{0} \tau_{0}}-\delta_{1}(x) \varepsilon, & x \in[c, 1],\end{cases} \\
& \Psi(x)= \begin{cases}\varphi(x)+\gamma_{1} \varepsilon, & x \in[0, c], \\
\psi(x)+\gamma_{2} \varepsilon e^{-\sigma_{0} \tau_{0}}+\delta_{1}(x) \varepsilon, & x \in[c, 1],\end{cases}
\end{aligned}
$$

where $\gamma_{1}, \gamma_{2}$ are positive constants such that

$$
\sigma_{0} \gamma_{1}=\left(\sigma_{0}+1\right) \gamma_{2}+2, \quad \sigma_{0} \gamma_{2}>\left|\beta+\varphi^{\prime}(c)-\psi^{\prime}(c)\right|,
$$

and

$$
\delta(x)=\frac{2+\gamma_{2}}{\sigma_{0}}\left[2 e^{\frac{M(c-x)}{\sigma_{0}}}-1\right],
$$

which is a solution of the differential equation

$$
\sigma_{0} \delta^{\prime}+M \delta+\left(\gamma_{2}+2\right) M=0 .
$$

It follows from the construction of $\Phi$ and $\Psi$ that

$$
\begin{aligned}
& \Phi(0)<0<\Psi(0), \\
& \Phi(1)<0<\Psi(1), \\
& {[\Phi](c)=[\Psi](c)=\alpha,} \\
& {\left[\Phi^{\prime}\right](c)>\beta>\left[\Psi^{\prime}\right](c) .}
\end{aligned}
$$

In what follows, we check the inequality (3). Using (H1) and (13) we have for $x \in(0, c)$,

$$
\begin{aligned}
& \Phi^{\prime \prime}(x)-g(x, \Phi(x)) \Phi^{\prime}(x)-f(x, \Phi(x)) \\
& \quad=\varphi^{\prime \prime}-g\left(x, \varphi(x)-\gamma_{1} \varepsilon\right) \varphi^{\prime}-f\left(x, \varphi(x)-\gamma_{1} \varepsilon\right) \\
& \quad \geq\left[g_{y}^{\prime}\left(x, \varphi(x)-\theta_{1} \gamma_{1} \varepsilon\right) \varphi^{\prime}+v\right] \gamma_{1} \varepsilon \\
& \quad>0,
\end{aligned}
$$


and for $x \in(c, 1)$,

$$
\begin{aligned}
\varepsilon & \Phi^{\prime \prime}(x)-g(x, \Phi(x)) \Phi^{\prime}(x)-f(x, \Phi(x)) \\
& =\varepsilon \psi^{\prime \prime}-\gamma_{1} \sigma_{0}^{2} e^{-\sigma_{0} \tau_{0}}-\delta^{\prime \prime} \varepsilon^{2}-g(x, \Phi(x))\left(\psi^{\prime}+\gamma_{1} \sigma_{0} e^{-\sigma_{0} \tau_{0}}-\delta^{\prime} \varepsilon\right)-f(x, \Phi(x)) \\
& \geq \varepsilon \psi^{\prime \prime}-\delta^{\prime \prime} \varepsilon^{2}-\sigma_{0} \delta^{\prime} \varepsilon+\left[g_{y}^{\prime}(x, \cdot) \psi^{\prime}+f_{y}^{\prime}(x, \cdot \cdot)\right]\left(\gamma_{2} e^{-\sigma_{0} \tau_{0}}+\delta_{1}\right) \varepsilon \\
& \geq\left(M-\delta^{\prime \prime} \varepsilon\right) \varepsilon-\left[\sigma_{0} \delta^{\prime}+M \delta+\left(\gamma_{2}+2\right) M\right] \varepsilon \\
& >0
\end{aligned}
$$

provided that $\varepsilon$ is small enough, where $(x, \cdot)=\left(x, \psi(x)-\theta_{2} \gamma_{2} \varepsilon e^{-\sigma_{0} \tau}-\theta_{2} \delta_{1} \varepsilon\right),(x, .)=$. $\left(x, \psi(x)-\theta_{3} \gamma_{2} \varepsilon e^{-\sigma_{0} \tau_{0}}-\theta_{3} \delta_{1} \varepsilon\right)$ and $0<\theta_{1}, \theta_{2}, \theta_{3}<1$.

For sufficiently small $\varepsilon>0$ the inequality (6) can be verified in a similar way. Thus we have proved that $\Phi(x)$ and $\Psi(x)$ are lower and upper solutions of (2), respectively. The conclusion immediately follows from Lemma 1.

Case (II). Assume ( $\left.\mathrm{H} 2^{\prime}\right)$.

In this case, the solution to the right problem (11) exhibits a boundary layer at $x=1$. Hence, we need first to establish a solution of the left problem. Considering the interface conditions we impose the following nonlinear boundary condition:

$$
g(c, y(c)+\alpha)\left(y^{\prime}(c)+\beta\right)+f(c, y(c)+\alpha)=0
$$

at $x=c$ for the left problem. We have the following proposition.

Proposition 2 Assume that

$$
g_{y}^{\prime}(x, y) \beta+f_{y}^{\prime}(x, y) \geq \bar{v}>0, \quad(x, y) \in(0, c) \times \mathbb{R} .
$$

Then the left boundary value problem

$$
\left\{\begin{array}{l}
y^{\prime \prime}=g(x, y) y^{\prime}+f(x, y), \quad x \in(0, c), \\
y(0)=0, \quad q\left(y(c), y^{\prime}(c)\right)=0
\end{array}\right.
$$

has a solution $y=\bar{\varphi}(x) \in C^{2}[0, c]$, where $q(u, v)=-g(c, u+\alpha)(v+\beta)-f(c, u+\alpha)$.

Proof The conclusion follows from Theorem 3.2 in [13], by verifying that $\Phi=-\bar{C}_{0}$ and $\Psi=\bar{C}_{0}$ are a pair of lower and upper solutions of (15), where

$$
\bar{C}_{0}=\max \left\{\frac{|f(x, 0)|}{v}, \frac{|f(c, \alpha)+g(c, \alpha) \beta|}{\bar{v}}\right\} .
$$

$\left(\mathrm{H}^{\prime}\right)$ Assume that the right reduced problem

$$
\left\{\begin{array}{l}
0=g(x, y) y^{\prime}+f(x, y), \quad x \in(c, 1), \\
y(c)=\bar{\varphi}(c)+\alpha
\end{array}\right.
$$

has a solution $y=\bar{\psi}(x) \in C^{2}[c, 1]$. 
In general, $\bar{\psi}(1) \neq 0$, and thereby we need to construct a corrected boundary layer term. To this end, substituting

$$
y=\bar{\psi}(x)+v(\tau), \quad \tau=\frac{x-1}{\varepsilon}
$$

into the right boundary value problem

$$
\left\{\begin{array}{l}
\varepsilon y^{\prime \prime}=g(x, y) y^{\prime}+f(x, y), \quad x \in(c, 1) \\
y(c)=\bar{\varphi}(c)+\alpha, \quad y(1)=0
\end{array}\right.
$$

and letting $\varepsilon \rightarrow 0$, we obtain

$$
\left\{\begin{array}{l}
\frac{d^{2} v}{d \tau^{2}}=g(1, \bar{\psi}(1)+v(\tau)) \frac{d v}{d \tau} \\
v(0)=-\bar{\psi}(1), \quad v\left(\frac{c-1}{\varepsilon}\right)=0
\end{array}\right.
$$

Considering the continuity of $g(x, y)$ we introduce

$$
\sigma_{2}=\max \{g(x, \bar{\psi}(x)+v):-|\bar{\psi}(1)| \leq v \leq|\bar{\psi}(1)|\} .
$$

The following proposition concerns the asymptotic behavior of the boundary layer term, whose proof is substantially similar to that of Lemma 3.1 in [14].

Proposition 3 The boundary value problem (17) has a solution $v=v(\tau)$ with the exponential estimates

$$
-\bar{\psi}(1) e^{\underline{\sigma} \tau} \leq v(\tau) \leq-\bar{\psi}(1) e^{\bar{\sigma} \tau}, \quad\left|\frac{d \nu}{d \tau}\right| \leq \sigma_{2}|\bar{\psi}(1)| e^{\sigma_{1} \tau},
$$

where

$$
\underline{\sigma}=\left\{\begin{array}{ll}
\sigma_{1}, & \text { if } \bar{\psi}(1)>0, \\
\sigma_{2}, & \text { if } \bar{\psi}(1)<0,
\end{array} \quad \bar{\sigma}= \begin{cases}\sigma_{2}, & \text { if } \bar{\psi}(1)>0, \\
\sigma_{1}, & \text { if } \bar{\psi}(1)<0 .\end{cases}\right.
$$

Theorem 2 Let the conditions (H1), ( $\left.\mathrm{H}^{\prime}\right),\left(\mathrm{H} 3^{\prime}\right)$, and (14) hold. Moreover, we assume that

$$
g_{y}^{\prime}(x, \bar{\varphi}(x)) \bar{\varphi}^{\prime}(x) \geq 0, \quad x \in(0, c) .
$$

Then for sufficiently small $\varepsilon>0$ the boundary value problem (2) has a solution $y(x, \varepsilon)$ such that for $x \in[0, c]$

$$
y(x, \varepsilon)=\bar{\varphi}(x)+\mathcal{O}(\varepsilon)
$$

and for $x \in[c, 1]$

$$
-\varrho \varepsilon-\bar{\psi}(1) e^{\frac{\sigma}{-\frac{x-c}{\varepsilon}}} \leq y(x, \varepsilon)-\bar{\psi}(x) \leq-\bar{\psi}(1) e^{\bar{\sigma} \frac{x-c}{\varepsilon}}+\varrho \varepsilon,
$$

where $\underline{\sigma}$ and $\bar{\sigma}$ are defined in Proposition 3, and $\varrho>0$ is a constant independent of $\varepsilon$. 
Proof It follows from the assumptions (H1) and (H3') that there exists a positive constant $\bar{M}$ such that for sufficiently small $\varepsilon>0$

$$
\begin{aligned}
& \left|\bar{\psi}^{\prime \prime}(x)\right| \leq \bar{M}, \quad\left|g_{y}^{\prime}(x, y) \bar{\psi}^{\prime}(x)\right| \leq \bar{M}, \\
& \left|g_{y}^{\prime}(x, y) \bar{\psi}^{\prime}(x) \bar{\psi}(1)\right| \leq \bar{M}, \quad\left|f_{y}^{\prime}(x, y) \bar{\psi}(1)\right| \leq \bar{M},
\end{aligned}
$$

where $(x, y) \in[c, 1] \times[\vartheta-\bar{M} \varepsilon, \vartheta+\bar{M} \varepsilon]$, and $\vartheta=\max \{|\bar{\psi}(x)|+|\bar{\psi}(1)|, c \leq x \leq 1\}$.

Select the bounding functions as follows:

$$
\begin{aligned}
& \Phi(x)= \begin{cases}\bar{\varphi}(x)-\left(\bar{\gamma}_{1} x+\bar{\gamma}_{2}\right) \varepsilon, & x \in[0, c], \\
\bar{\psi}(x)-\bar{\psi}(1) e^{\underline{\sigma} \tau}-\bar{v}(\tau) \varepsilon-\bar{\delta}(x) \varepsilon, & x \in[c, 1],\end{cases} \\
& \Psi(x)= \begin{cases}\bar{\varphi}(x)+\left(\bar{\gamma}_{1} x+\bar{\gamma}_{2}\right) \varepsilon, & x \in[0, c], \\
\bar{\psi}(x)-\bar{\psi}(1) e^{\underline{\sigma} \tau}+\bar{v}(\tau) \varepsilon+\bar{\delta}(x) \varepsilon, & x \in[c, 1],\end{cases}
\end{aligned}
$$

where $\bar{\gamma}_{1}, \bar{\gamma}_{2}$ are positive constants which can be chosen such that (4) and (7) hold. The function

$$
\bar{\delta}(x)=\frac{L}{\bar{M}}\left(e^{\frac{\bar{M}}{\sigma_{1}} x}-1\right), \quad L>\bar{M}^{3}+\bar{M}
$$

is a solution of the equation

$$
\sigma_{1} \bar{\delta}^{\prime}-\overline{M \delta}=L
$$

and the function

$$
\bar{v}=\frac{\bar{M}\left(\bar{M} \sigma_{1}-2 \tau\right) e^{\sigma_{1} \tau}}{\sigma_{1}}
$$

solves

$$
\frac{d^{2} \bar{v}}{d \tau^{2}}-\sigma_{1} \frac{d \bar{v}}{d \tau}+2 \bar{M} e^{\sigma_{1} \tau}=0, \quad \bar{v}(0)=\bar{M}^{2},
$$

and

$$
\frac{d \bar{\nu}}{d \tau}=\frac{\bar{M}\left(\bar{M} \sigma_{1}^{2}-2 \sigma_{1} \tau-2\right) e^{\sigma_{1} \tau}}{\sigma_{1}}>0, \quad \text { for } \tau<0
$$

Here we check the inequality (3) only for $x \in(c, 1)$, since the equality on $x \in(0, c)$ can be verified by following similar lines as in the proof of Theorem 1 . From the definition of $\Phi(x)$ we have for $x \in(c, 1)$

$$
\begin{aligned}
& \varepsilon \Phi^{\prime \prime}(x)-g(x, \Phi(x)) \Phi^{\prime}(x)-f(x, \Phi(x)) \\
& =\varepsilon \bar{\psi}^{\prime \prime}-\bar{\delta}^{\prime \prime} \varepsilon^{2}-\frac{\bar{\psi}(1) \underline{\sigma}^{2} e^{\underline{\sigma} \tau}}{\varepsilon}-\frac{d^{2} \bar{v}}{d \tau^{2}}-g(x, \Phi)\left(\bar{\psi}^{\prime}-\frac{\bar{\psi}(1) \underline{\sigma} e^{\underline{\sigma} \tau}}{\varepsilon}-\frac{d \bar{v}}{d \tau}-\bar{\delta}^{\prime} \varepsilon\right)-f(x, \Phi) \\
& =\varepsilon \bar{\psi}^{\prime \prime}-\bar{\delta}^{\prime \prime} \varepsilon^{2}-\frac{d^{2} \bar{v}}{d \tau^{2}}+g(x, \Phi) \frac{d \bar{v}}{d \tau}-\frac{\bar{\psi}(1) \underline{\sigma}}{\varepsilon}[\underline{\sigma}-g(x, \Phi)] e^{\underline{\sigma} \tau}+g(x, \Phi) \bar{\delta}^{\prime} \varepsilon
\end{aligned}
$$




$$
\begin{aligned}
& +\left[g(x, \bar{\psi})-g\left(x, \bar{\psi}-\bar{\psi}(1) e^{\underline{\sigma} \tau}\right)\right] \bar{\psi}^{\prime}+\left[g\left(x, \bar{\psi}-\bar{\psi}(1) e^{\underline{\sigma} \tau}\right)-g(x, \Phi)\right] \bar{\psi}^{\prime} \\
& +\left[f(x, \bar{\psi})-f\left(x, \bar{\psi}-\bar{\psi}(1) e^{\underline{\sigma} \tau}\right)\right]+\left[f\left(x, \bar{\psi}-\bar{\psi}(1) e^{\underline{\sigma} \tau}\right)-f(x, \Phi)\right] \\
\geq & -\bar{M} \varepsilon-\bar{\delta}^{\prime \prime} \varepsilon^{2}-\frac{d^{2} \bar{v}}{d \tau^{2}}+\sigma_{1} \frac{d \bar{v}}{d \tau}-2 \bar{M} e^{\sigma_{1} \tau}-\bar{M}(\bar{v}+\bar{\delta}) \varepsilon+\nu(\bar{v}+\bar{\delta}) \varepsilon+\sigma_{1} \bar{\delta}^{\prime} \varepsilon \\
\geq & -\bar{M} \varepsilon-\bar{\delta}^{\prime \prime} \varepsilon^{2}-\bar{M} \bar{\nu} \varepsilon+\left(\sigma_{1} \bar{\delta}^{\prime}-\overline{M \delta}\right) \varepsilon \\
\geq & \left(L-M-M^{3}-\delta^{\prime \prime} \varepsilon\right) \varepsilon \\
> & 0
\end{aligned}
$$

on condition that $\varepsilon$ is sufficiently small. Thus $\Phi(x)$ is a lower solution of (2).

It can be shown similarly that $\Psi(x)$ is an upper solution of (2). From Lemma 1 it follows that there is a solution with the estimates (19) and (20).

Finally, as an illustration, let us consider a linear interface boundary value problem,

$$
\left\{\begin{array}{l}
\lambda_{\varepsilon} y^{\prime \prime}=y^{\prime}+2 y, \quad x \in(0,0.5) \cup(0.5,1) \\
y(0)=y(1)=0, \\
{[y](0.5)=0, \quad\left[y^{\prime}\right](0.5)=1}
\end{array}\right.
$$

From Theorem 2 it follows that (21) has a solution with the following asymptotic estimate:

$$
y(x, \varepsilon)= \begin{cases}\frac{e^{-x}-e^{2 x}}{4 e-e^{-\frac{1}{2}}}+\mathcal{O}(\varepsilon), & x \in[0,0.5], \\ \frac{e^{x-\frac{1}{2}}-e^{2(1-x)}}{4 e-e^{-\frac{1}{2}}}+\frac{e-e^{-\frac{3}{2}}}{4 e-e^{-\frac{1}{2}}} e^{\frac{x-1}{\varepsilon}}+\mathcal{O}(\varepsilon), & x \in[0.5,1]\end{cases}
$$

which agrees with the exact solution accurate to order $\varepsilon$.

\section{Competing interests}

The author declares that he has no competing interests.

\section{Author's contributions}

The author read and approved the final manuscript.

\section{Acknowledgements}

The author wants to thank the referees for valuable comments and suggestions. The author was supported by the National Natural Science Foundation of China (No. 11371087), in part by the Natural Science Foundation of Shanghai (No. 12ZR1400100), and by the Fundamental Research Funds for the Central Universities.

Received: 3 July 2014 Accepted: 13 August 2014 Published online: 25 September 2014

\section{References}

1. de Falco, C, O'Riordan, E: Interior layers in a reaction-diffusion equation with a discontinuous diffusion coefficient. Int. J. Numer. Anal. Model. 7, 444-461 (2010)

2. Aitbayev, R: Existence and uniqueness for a two-point interface boundary value problems. Electron. J. Differ. Equ. 2013, $242(2013)$

3. Chern, I, Shu, Y: A coupling interface method for elliptic interface problems. J. Comput. Phys. 225, $2138-2174$ (2007)

4. Loubenets, A, Ali, T, Hanke, M: Highly accurate finite element method for one-dimensional elliptic interface problems. Appl. Numer. Math. 59, 119-134 (2009)

5. Huang, Z: Tailored finite point method for the interface problem. Netw. Heterog. Media 4, 91-106 (2009)

6. Aguilar, G, Lisbona, F: Singular perturbation on a subdomain. J. Math. Anal. Appl. 210, 292-307 (1997)

7. Aguilar, G, Lisbona, F: On the coupling of elliptic and hyperbolic nonlinear differential equations. Math. Model. Numer. Anal. 28(4), 399-417 (1994)

8. Coster, CD, Habets, P: Two-Point Boundary Value Problems: Lower and Upper Solutions. Elsevier, New York (2006)

9. de Jager, EM, Jiang, F: The Theory of Singular Perturbations. North-Holland, Amsterdam (1996)

10. O'Malley, RE: Singular Perturbation Methods for Ordinary Differential Equations. Springer, New York (1991)

11. Zeidler, E: Applied Functional Analysis: Applications to Mathematical Physics. Springer, New York (1995) 
12. Kelley, WG, Peterson, AC: The Theory of Differential Equations. Springer, New York (2010)

13. Fabry, C, Habets, P: Upper and lower solutions for second-order boundary value problems with nonlinear boundary conditions. Nonlinear Anal. TMA 10, 985-1007 (1986)

14. Xie, F: On a class of singular boundary value problems with singular perturbation. J. Differ. Equ. 252, 2370-2387 (2012)

doi:10.1186/s13661-014-0201-8

Cite this article as: Xie: An interface problem with singular perturbation on a subinterval. Boundary Value Problems 2014 2014:201

Submit your manuscript to a SpringerOpen ${ }^{\circ}$ journal and benefit from:

- Convenient online submission

- Rigorous peer review

- Immediate publication on acceptance

Open access: articles freely available online

- High visibility within the field

- Retaining the copyright to your article

Submit your next manuscript at $>$ springeropen.com 